THU0345

EFFECT OF THE LONG-TERM RITUXIMAB TREATMENT ON B-LYMPHOCYTES AND ANTINUCLEAR AUTOANTIBODY LEVEL IN PATIENTS WITH SYSTEMIC SCLEROSIS

L. Garzanova ${ }^{1}$, L. P. Ananyeva' ${ }^{1}$, O. Koneva ${ }^{1}$, O. Desinova ${ }^{1}$, O. Ovsyannikova ${ }^{1}$ M. Starovoytova ${ }^{1}$, S. Glukhova ${ }^{1}$, M. Cherkasova ${ }^{1}$, A. Aleksankin ${ }^{1} .{ }^{1}$ V.A. Nasonova Research Institute of Rheumatology, MOSCOW, Russian Federation

Background: Anti-B-cell therapy is seen as a promising therapeutic option for systemic sclerosis (SSc). The study of antinuclear antibody levels during treatment with rituximab (RTX) in patients (pts) with SSc could have theoretical and practical interest.

Objectives: To assess the changes in ANA, anti-topoisomerase-1 (Scl-70) levels and B-lymphocytes (B-lymph) count during RTX therapy during prospective observation.

Methods: This prospective study included 88 pts with SSc, $83 \%$ of them had interstitial lung disease and $75 \%$ had positive Scl-70 autoantibody. The mean age was 47 yrs (17-71), female- 73 pts $(83 \%)$, the diffuse cutaneous subset of the disease had $50 \mathrm{pts}(57 \%)$. The mean disease duration was $5,9 \pm 4,8 \mathrm{yrs}$ The mean follow-up period was 27 months (12-42). The cumulative mean dose of RTX was 2,9 $91,1 \mathrm{grams}$. All patients received prednisolone at a dose of $11,7 \pm 4,4 \mathrm{mg}$, immunosuppressants received $42 \%$ of them. Patients were divided into groups depending on the duration of the disease: group $1(n=33)$ - up to 3 yrs, group $2(n=25)$ - from 3 to 6 yrs, group $3(n=30)$ - more than 6 years (6-18yrs). The results are presented in the form of mean values, median, upper and lower quartiles.

Results: Parallel to clinical improvement in most patients (96\%) we found positive changes in many parameters at the end of the study compared to the baseline. The Rodnan skin score decreased from $11,21 \pm 9,33$ to $6,19 \pm 4,74$ $(p<0,001)$. The disease activity index (EScSG-AI) decreased from $2,9 \pm 1,74$ to $1,36 \pm 1,15(\mathrm{p}<0,001)$. Forced vital capacity, \% predicted, increased from $76,35 \pm 19,65$ to $84,37 \pm 21,04(p<0,001)$. Diffusing capacity for carbon monoxide, \% predicted, increased from $45,56 \pm 17,72$ to $47,62 \pm 16,96$ ( $p<0,019)$. The dose of prednisolone decreased from $11,7 \pm 4,4$ to $9,2 \pm 3,2 \mathrm{mg}(p<0,001)$. The absolute number of B-lymph decreased from $0,224 \pm 0,19$ to $0,0175 \pm 0,058$ $(p<0,001)$. The pts of the group 1 showed the highest values of B-lymph at baseline and level of B-lymph decreased from $0,326 \pm 0,22$ to $0,008 \pm 0,01(\Delta$ 0,318 ) at the end of the study. In group 2 depletion was less pronounced (from $0,197 \pm 0,14$ to $0,026 \pm 0,07(\Delta 0,171)$ and the lowest depletion was observed in group 3 (from $0,151 \pm 0,16$ to $0,019 \pm 0,07(\Delta 0,131), p<0,001$ for all groups. An initially positive ANA was found in $92 \%$ of pts (range 1/320-1/1280). During observation, the number of pts with high (1/640-1/1280) ANA titers decreased from 70 to $41(p<0,001)$, and the average level of ANA decreased by $30-40 \%$ in all groups. At baseline 63 pts $(75 \%)$, had positive Scl-70 with equal levels in all groups. At the end of the study level of Scl-70 decreased from 125,02 $\pm 89,12$ to $108,6 \pm 86,89$ units $/ \mathrm{ml}(\mathrm{p}<0,007)$. A negative correlation was found between the duration of the disease and ANA $(r=-0,54 ; p<0,003)$ and Scl-70 $(r=-0,44$; $\mathrm{p}<0,017)$.

Conclusion: In our study a clinical improvement was shown in most pts at the long-term complex therapy, including RTM. We found a significant decrease in the absolute number of B-lymph, as well as decrease of ANA and Scl-70 levels. Initially pts with a short duration of the disease had a higher level of B-lymph and in these pts depletion was more pronounced, compared to those with a longer duration of the disease. However, the level of Scl-70 and ANA decreased both to those who started RTX therapy at an early stage of the disease $(<3 y r s)$ and to those who had a long disease duration.

Disclosure of Interests: None declared

DOI: 10.1136/annrheumdis-2020-eular.2490

\section{THU0346 \\ SARC-F PERFORMANCE FOR SARCOPENIA SCREENING IN PATIENTS WITH SYSTEMIC SCLEROSIS}

V. $\operatorname{Hax}^{1}$, R. Santo ${ }^{1}$, L. Santos ${ }^{1}$, M. Farinon ${ }^{1}$, M. De Oliveira ${ }^{1}$, G. Levi Três ${ }^{1}$, A. A. Gasparin ${ }^{1}$, M. Bredemeier ${ }^{2}$, R. Xavier ${ }^{1}$, R. Mendonça Da Silva Chakr ${ }^{1}$. ${ }^{1}$ Hospital de Clínicas de Porto Alegre, Department of Rheumatology, Porto Alegre, Brazil; ${ }^{2}$ Hospital Nossa Senhora da Conceição, Department of Rheumatology, Porto Alegre, Brazil

Background: Because the method of diagnosing sarcopenia is complex and is considered to be difficult to introduce into routine practice, the European Working Group on Sarcopenia in Older People's (EWGSOP) recommends use of the SARC-F questionnaire as a way to introduce assessment and treatment of sarcopenia into clinical practice ${ }^{1}$. Only recently, some studies have focused their attention on the presence of sarcopenia in systemic sclerosis (SSc) and there is no data about the performance of SARC-F in this population.
Objectives: To test the diagnostic properties of the SARC-F questionnaire for sarcopenia screening in SSc patients.

Methods: Cross-sectional study, including 94 SSc patients assessed by clinical evaluation, laboratory and pulmonary function tests. Sarcopenia was evaluated using the EWGSOP diagnostic criteria updated in 2019 (EWGSOP2): dual-energy X-ray absorptiometry, handgrip strength, and short physical performance battery (SPPB) ${ }^{1}$. Participants also completed the SARC-F questionnaire. The questionnaires' performances were evaluated through receiver operating characteristic (ROC) curves and standard measures of diagnostic accuracy were computed using the EWGSOP2 criteria as the gold standard for diagnosis of sarcopenia

Results: Sarcopenia was identified in $15(15,9 \%)$ SSc patients by the EWGSOP2 criteria. Area under the ROC curve of SARC-F screening for sarcopenia was $0.588(95 \%$ confidence interval $(\mathrm{Cl}) 0.482,0.688)$ (figure 1). The results of sensitivity, specificity, positive likelihood ratio (PLR) and negative likelihood ratio (NLR) with the EWGSOP2 criteria as the reference standard were 35.71 [95\% Cl, 12.76-64.86], 81.01 (95\% Cl, 70.62-88.97), 1.88 (95\% $\mathrm{Cl}, 0.81-4.35)$ and $0.79(95 \% \mathrm{Cl}, 0.53-1.19)$, respectively. The optimal cut-off point of SARC-F in our sample was $\geq 4$ (Youden index: 0.21 ), the same cut-off point recommended in the literature ${ }^{2,3}$. Only $6(40 \%)$ out of the 15 participants with sarcopenia were identified by the SARC-F questionnaire in our population. However, the SARC-F properly identified 4 out of 5 patients who had severe sarcopenia.

Conclusion: This is the first study to evaluate the performance of SARC-F questionnaire for sarcopenia screening in patients with SSc. Although it appropriately identifies severe cases of sarcopenia, the SARC-F alone may not be an adequate screening tool in high-risk populations, such as SSc, that may benefit from early intervention and treatment.

References:

[1] Cruz-Jentoft AJ, Bahat G, Bauer J, et al. Sarcopenia: Revised European consensus on definition and diagnosis. Age Ageing. 2019:48(1):16-31.

[2] Malmstrom TK, Morley JE. SARC-F: A simple questionnaire to rapidly diagnose sarcopenia. J Am Med Dir Assoc. 2013;14(8):531-532.

[3] Ida S, Kaneko R, Murata K. SARC-F for Screening of Sarcopenia Among Older Adults: A Meta-analysis of Screening Test Accuracy. J Am Med Dir Assoc. 2018;19(8):685-689.

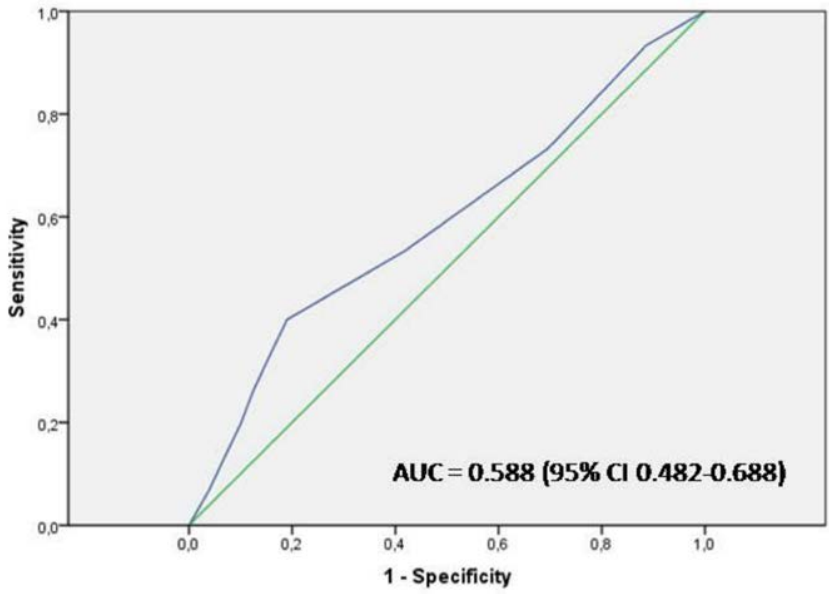

Figure 1. Receiver operating characteristic (ROC) curve analyze of SARC-F screening for sarcopenia ( $n=94$ ). Discrimination was quantified using the area under the curve (AUC). The optimal cut-off point of SARC-F was $\geq 4$.

Disclosure of Interests: Vanessa Hax: None declared, Rafaela Santo: None declared, Leonardo Santos: None declared, Mirian Farinon: None declared, Marianne de Oliveira: None declared, Guilherme Levi Três: None declared, Andrese Aline Gasparin: None declared, M Bredemeier: None declared, Ricardo Xavier Consultant of: AbbVie, Pfizer, Novartis, Janssen, Eli Lilly, Roche, Rafael Mendonça da Silva Chakr: None declared

DOI: 10.1136/annrheumdis-2020-eular.2241

\section{THU0347 \\ CLINICAL DESCRIPTION OF A COHORT OF PATIENTS WITH SCLEROTIC-TYPE CHRONIC GRAFT-VERSUS- HOST DISEASE TREATED IN A MULTIDISCIPLINARY PRACTICE}

C. Hidalgo ${ }^{1}$, C. Román ${ }^{2}$, M. E. Acosta ${ }^{3}$, L. Gómez-Lechón ${ }^{3}$, L. Pantoja ${ }^{4}$, M. D. Sánchez ${ }^{5}$, O. Compán ${ }^{1}$, S. Pastor ${ }^{1}$, C. A. Montilla-Morales ${ }^{1}$, L. López ${ }^{6}$. ${ }^{1}$ Hospital Universitario de Salamanca, Salamanca, Spain; ${ }^{2}$ Hospital 
Universitario de Salmanca, Salamanca, Spain; ${ }^{3}$ Hospital Universitario de Salamanca, Salamanca, Spain; ${ }^{4}$ Complejo Asistencial Hospital General de Segovia, Rheumatology, Segovia, Spain; ${ }^{5}$ Hospital Clínico Universitario de Valladolid, Rheumatology, Valladolid, Spain; ${ }^{6}$ Hospital Universitario de Salamanca, Haematology, Salamanca, Spain

Background: Graft versus host disease is the most frequent complication after allogeneic transplantation of hematopoietic progenitors. Its chronic form usually involves a multisystemic syndrome that reflects a complex immune response with varying degrees of inflammation, immune dysregulation and fibrosis, responsible for the characteristic clinical manifestations of the disease. Joint, muscular and fascial involvement represents one of the areas, often unnoticed or poorly evaluated, that negatively impacts the physical function and quality of life of these patients.

Objectives: Describe the presence of musculoskeletal manifestations and their clinical characteristics in patients with chronic GVHD (cGVHD) evaluated in a multidisciplinary consultation

Methods: Descriptive and retrospective observational study to detail the initial presence and during the follow-up of diagnostic and nonspecific musculoskeletal manifestations of cGVHD in a cohort of 103 patients included in the database. The clinical characteristics of 68 patients with a defined diagnosis of sclerotic cGVHD are described. Demographic variables are collected along with clinical conditions about transplant and in a systematic way the assessment according to diagnostic criteria of the American National Institute of Health 2015 highlighting: range of motion scale and photographic range of motion ( $P-R O M)$ scale in shoulders, elbows, hands and ankles; and laboratory data: presence of eosinophilia and autoantibodies. Descriptive and frequency statistical analysis was done using Microsoft Office Excel 2007.

Results: Sixty-eight(66\%)patients meet diagnostic criteria for sclerotic cGVHD during follow-up. Forty-five(66.2\%) women and $23(33.8 \%)$ men, with a mean age of 54.5 years (range 10-78), Acute myeloid leukemia was the reason for transplant in $20(29,4 \%)$ followed by non-Hodgkin lymphoma in 15 (16.2\%). In 40(58.7\%) patients it was performed from a related donor and with reduced intensity conditioning in $43(63.2 \%)$. Only in one patient the source of hematopoietic progenitors was bone marrow (rest peripheral blood). The average time from transplant to the first visit was 29.5 months (range 4 -168). Twelve(17.64\%) patients presented isolated joint/fascial involvement without objective skin involvement (Table 1)

Table 1. Clinical features of the sclerotic joint/fascial chronic GVHD cohort $(\mathrm{N}=68)$

\begin{tabular}{ll}
\hline Unspecific musculoskeletal symptoms: & $46(67.6 \%)$ \\
-Artromyalgia & $39(57.3 \%)$ \\
-Edema & $13(19 \%)$ \\
-Stiffness & $4(5.8 \%)$ \\
Fasciitis/contractures & $28(41.2 \%) / 43(63,2 \%)$ \\
Restricted ROM ${ }^{1}$ & $34(50 \%) / 14(20.2 \%) / 4(5.8 \%)$ \\
-Mild/Moderate/Severe & \\
Impaired mobility & $26(38.2 \%)$ \\
-Shoulders & $21(30.8 \%)$ \\
-Elbows & $29(42.6 \%)$ \\
-Wrist/fingers & $21(30.6 \%)$ \\
-Ankles & \\
Sclerodermatous involvement & $6(8.8 \%) / 7(10.3 \%)$ \\
-Superficial /deep & $20(29.4 \%)$ \\
-Mixed (sclerodermiform + lichenoid) & $13(19.2)$ \\
- Overlapping skin sclerosis & $25(36.7 \%) / 7(10.3 \%) / 1(1.5 \%)$ \\
NIH ${ }^{1}$ Global Score & \\
-Mild/Moderate/Severe & $21(30.8 \%)$ \\
Eosinophilia & $14(20.6 \%)$ \\
Autoantibodies &
\end{tabular}

${ }^{1} \mathrm{ROM}$ (range of motion) ${ }^{2} \mathrm{NIH}$ : National Institute of Health

Conclusion: Joint involvement secondary to sclerosis is very common in our cohort, mainly of the dorsal wrist flexion with deleterious repercussion on physical function. It needs to be recognized and evaluated early with validated scales. The search for new biomarkers associated with fibrosis, the use of advanced imaging techniques and the multidisciplinary approach can help improve the prognosis of patients with cGVHD.

References:

[1] Jagasia MH,Blood Marrow Transplant. 2015;21(3):389-40

Disclosure of Interests: None declared

DOI: 10.1136/annrheumdis-2020-eular.3386

\section{THU0348 1 ALTERED IMMUNE RECOGNITION OF SPECIFIC GUT BACTERIA BY IMMUNOGLOBULINS IN EARLY SYSTEMIC SCLEROSIS}

A. M. Hoffmann-Vold ${ }^{1}$, K. Andréasson ${ }^{2}$, S. Hyll Hansen ${ }^{1}$,

S. Midtvedt ${ }^{3}$, H. Fretheim ${ }^{1}$, H. Didriksen ${ }^{1}$, T. Garen ${ }^{1}$, E. Bækkevold ${ }^{1}$,

$\varnothing$. Midtvedt ${ }^{1}$, R. Hesselstrand ${ }^{2}$, B. K. Chung ${ }^{1}, \varnothing$. Molberg ${ }^{1} .{ }^{1}$ Oslo University
Hospital, Oslo, Norway; ${ }^{2}$ Lund University Hospital, Lund, Sweden; ${ }^{3}$ NTNU, Trondheim, Norway

Background: Gastrointestinal tract (GIT) involvement is highly prevalent in systemic sclerosis (SSc) and associates with GIT symptoms that are present early and progress over time. Changes in gut microbiota are often reported in inflammatory disease settings but whether GIT symptoms associate with altered immune recognition of specific gut bacteria in early SSc is unknown.

Objectives: Here, we profiled Ig coating patterns of gut bacteria in early disease from two well-characterized SSc cohorts to determine if the pattern and extent of bacterial immunoglobulin (Ig) coating differs in early SSc.

Methods: We collected fecal material from early SSc patients ( $<36$ months from time of diagnosis) at Oslo and Lund University Hospitals and from healthy age and gender matched controls ( $\mathrm{HC})$. To assess whether adaptive immunity was triggered against gut microbiota in early disease, we sorted and sequenced $\lg \mathrm{A}$ IgM and IgG coated bacteria from fecal samples by flow cytometry and performed 16s rRNA sequencing to compare the relative Ig coating of early SSc patients to HC. Data was resolved to the family level, rarefied to 5101 reads and converted to relative abundance. Taxonomic profiles, relative abundance, $\lg \mathrm{A}$ $\operatorname{lgM}$ and IgG coating patterns and extent of Ig coating were assessed. Unadjusted $p$-values $<0.05$ were defined as significant.

Results: We included 50 SSc patients (26 from Oslo, 24 from Lund) with early SSc and 9 gender and age matched HC. Mean age of SSc patients at time of inclusion was 53 years, mean time since diagnosis was 13 months; $82 \%$ were female, $61 \%$ had limited cutaneous SSc and $43 \%$ were anti-centromere antibody positive. In all, $82 \%$ were treatment naïve while $18 \%$ had received either cyclophosphomide or mycophenolate mofetil immunosuppressants. We found increased relative abundance of IgA coated Desulfovibrionaceae in both SSc cohorts compared to $\mathrm{HC}$ and increased IgM and IgG coating of Veillonellaceae and Streptococcaceae (Figure 1). All of these bacteria have previously been associated with other autoimmune diseases or pro-inflammatory status: Desulfovibrionaceae to immune activation in the gut, and Veillonellaceae and Streptococcaceae to other chronic inflammatory and fibrotic conditions. While abundance of $\lg$ A coated Desulfovibrionaceae was higher in cyclophosphomide or mycophenolate mofetil-treated SSc patients than untreated patients, Veillonellaceae and Streptococcaceae were not affected by treatment. A lower abundance of IgA and IgM coated Akkermansiaceae; and IgM and IgG coated Bifidobacteriaceae was detected in treated compared to treatment naïve early SSc patients (Figure 2).

Figure 1: Phylogenetictree of gut bacteria based on the 165 rRNA gene sequence showing relative abundance of $\lg A$, Ig Helo

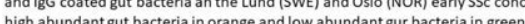
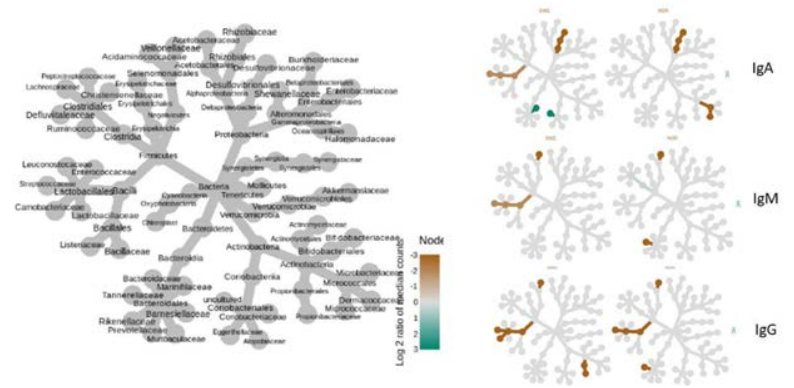

Figure 2: Phylogenetictree of gut bacteria based on the 165 rRNA gene sequence showing relative abundance of $\operatorname{lgA}, \lg M$ and IgG coated gut bacteria in treated (yes) and untreated (no) SSC patients with early disease with high abundant gut bacteria in orange and low abundant gur bacteria in green

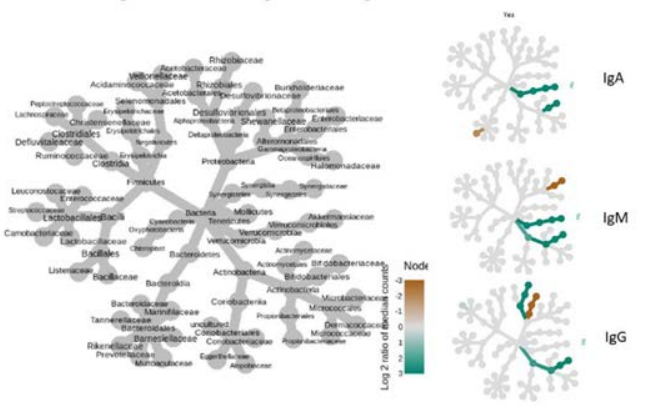

Conclusion: We find the pattern and extent of Ig coating to inflammatory-associated gut bacteria differs between treatment-naïve, early SSc patients treated with cyclophosphomide or mycophenolate mofetil and $\mathrm{HC}$ which suggests 